\title{
Determinación del Contenido de Polifenoles Totales, Flavonoides y Actividad Antioxidante de 34 Cafés Comerciales de Panamá
}

\author{
Aracelly Vega*, Javier A. De León y Stephany M. Reyes \\ Centro de Investigación en Recursos Naturales, Universidad Autónoma de Chiriquí. Apartado Postal 0427, \\ Chiriquí, República de Panamá (e-mail: aravega@cwpanama.net).
}

${ }^{*}$ Autor a quien debe ser dirigida la correspondencia

Recibido Dic. 9, 2016; Aceptado Feb. 22, 2017; Versión final Abr. 20, 2017, Publicado Ago. 2017

\begin{abstract}
Resumen
El objetivo de esta investigación fue determinar el contenido de polifenoles totales, flavonoides y la actividad antioxidante de 34 cafés comerciales de Panamá. El café es una de las bebidas más populares en el mundo y por su contenido de sustancias bioactivas se le considera que tiene propiedades nutraceúticas. Los parámetros indicados fueron determinados espectrofotométricamente. El contenido de polifenoles totales de las muestras de cafés puros y mezclados estuvo en el rango de 28.60 a 46.82 y 11.17 a $16.10 \mathrm{mg} \mathrm{GAE} / \mathrm{g}$, respectivamente. El contenido de flavonoides fue de 22.16 a 38.29 y 9.36 a 14.92 $\mathrm{mg}$ equivalentes de catequina/g, respectivamente y la actividad antioxidante estuvo en el rango de $0.11 \mathrm{a}$ 0.20 y 0.025 a $0.061 \mathrm{mmol}$ trolox $(\mathrm{TE}) / \mathrm{g}$, respectivamente. Se encontró una correlación de $R^{2}=0.69$ entre actividad antioxidante y polifenoles totales para cafés puros y un $R^{2}=0.04$ para cafés mezclados. Estos resultados permiten concluir que los de polifenoles totales son componentes que aportan un porcentaje importante de la capacidad antioxidante del café.
\end{abstract}

\section{Determination of the Content of Total Polyphenols, Flavonoids and Antioxidant Activity in 34 Commercial Coffee Brands of Panama}

\begin{abstract}
The main objective of this research was to determine the content of total polyphenols, flavonoids and antioxidant activity of 34 commercial coffees in Panama. Coffee is one of the most popular beverages in the world, and it is considered to have nutraceutical properties due to its content of bioactive substances. The parameters of interest in this study were determined spectrophotometrically. Total polyphenols content of pure and mixed coffees was in the range of 28.60 to 46.82 and 11.17 to $16.10 \mathrm{mg}$ of gallic acid (GAE)/g of coffee respectively. Flavonoids content was 22.16 to 38.29 and 9.36 to $14.92 \mathrm{mg}$ of catechin/g of coffee respectively, and antioxidant activity was in the range of 0.11 to 0.20 and 0.025 to $0.061 \mathrm{mmol}$ of trolox $(\mathrm{TE}) / \mathrm{g}$ of coffee respectively. A correlation of $\mathrm{R}^{2}=0.69$ was found between antioxidant activity and total polyphenols for pure coffees and a correlation of $R^{2}=0.04$ for mixed coffees. These results allow concluding that total polyphenols are substances that importantly contribute to the antioxidant capacity of coffee.
\end{abstract}




\section{INTRODUCCIÓN}

El café es una de las bebidas más populares en el mundo y su consumo aumenta cada día (Esquivel y Jiménez, 2012; Nam, 2015). Inicialmente, por su contenido de cafeína y consiguientes propiedades energizantes y estimulantes (Wanyika, 2010), el café fue utilizado en todo tipo de bebidas y otros productos. En los últimos años, se han llevado a cabo estudios exhaustivos sobre el contenido de sustancias bioactivas y propiedades nutraceúticas del café (Farah, 2012), y por sus beneficios en prevenir enfermedades, se le clasifica como una bebida funcional. La calidad de la "taza" y el contenido de sustancias bioactivas del café natural depende de un gran número de factores, como la variedad (Martín et al., 1998; Vignoli et al., 2011; Hecimovic et al., 2011; Pérez-Hernández et al., 2012), condiciones ambientales (Guyot et al., 1996; Decazy et al., 2003), la tecnología del procesado (Parras et al., 2007) y la forma comercial como se ofrecen (Ludwing et al., 2014).

A nivel mundial, la tendencia es la producción de dos especies de café: Coffea arabica (café arábica), con casi $2 / 3$ de la producción mundial y la especie Coffea canephora (café robusta) con 1/3 de la producción mundial. En Panamá, estas dos especies se cultivan con fines comerciales. De la especie $C$. arabica, se cultivan las variedades Typica, Caturra Rojo, Borbón, Mundo Novo, MIDA 96 y Catuai; la mayoría, para exportación. Y de la especie C. canephora, las variedades Caracolillo y Robusta, para consumo nacional, mezclada con Arábica. También hay cafés especiales que se cultivan en pequeñas cantidades, como las variedades Geisha (Coffea arabica, var. geisha) y Pacamara (Coffea arabica, var. pacamara), ambas para exportación (Miranda, 2007). En Panamá, igual que en otros países, existen marcas de cafés comerciales que ofrecen el café en grano tostado o molido. En algunos casos, se mezclan con otros granos como maíz y frijol (ver Tabla 2). Y en muchas ocasiones, estas marcas no indican en sus etiquetas el contenido, ni la mezcla de sus cafés (Vega, 2007; Gallignani et al., 2008; Vega et al., 2014). En el mercado nacional, se venden en presentaciones de 20 a $30 \mathrm{~g}$ por paquete. También hay presentaciones de $220 \mathrm{~g}, 350 \mathrm{~g}, 425 \mathrm{~g}$ y $454 \mathrm{~g}$.

El grano de café contiene diferentes sustancias bioactivas, y las cantidades de estas sustancias en el extracto de café, variarán dependiendo de la técnica de extracción utilizada (Kreicbergs y Dimins, 2011; Kocadagli y Gökmen, 2016; Shang et al., 2017). Si deseamos conocer el contenido real de sustancias bioactivas en una taza de café y así saber lo que el ser humano ingiere, se debe extraer dichas sustancias con agua caliente, que es el método tradicional de preparación del café para consumo (Ludwing et al., 2014). Panamá al ser un país con baja producción de café a nivel mundial, de $7570202.3 \mathrm{~kg}$ de café pilado, cosecha de 2015 (MIDA, 2015), ha tenido que desarrollar estrategias para competir internacionalmente y vender su producto a buenos precios. Ha habido 19 reuniones anuales de Catación Internacional de Café en Panamá, en las cuales se ha reconocido la calidad de estos cafés por sus propiedades organolépticas. El café geisha, es un ejemplo de esto, ya que por años consecutivos ha ganado el primer lugar de los cafés especiales por sus propiedades organolépticas.

El café es una fuente considerable de polifenoles y compuestos fenólicos, que puede aportar antioxidantes a la dieta, particularmente en el caso de nuestro país, donde no se consumen de forma regular otras bebidas como el vino o el té, también abundantes en compuestos de esta naturaleza. En Svilaas et al. (2004) se reportó que el café es la fuente dietaria principal de antioxidantes, más que el vino y los vegetales y esto se debe a que a pesar de que existen alimentos con una mayor cantidad de antioxidantes que el café, éste se consume con más frecuencia y mayor volumen. En la actualidad no hay reporte sobre el contenido de antioxidantes de los cafés comerciales de Panamá, información importante para saber cuál es la ingesta de antioxidantes por taza de café. En Shang et al. (2017) se reportó el contenido de polifenoles del residuo sólido del café geisha de Panamá, pero no del grano.

El objetivo de este estudio fue determinar el contenido de polifenoles totales, flavonoides y la actividad antioxidante de 34 marcas de cafés comerciales de Panamá. Determinar la correlación entre la actividad antioxidante y el contenido de polifenoles totales de las marcas de café agrupadas en cafés puros y cafés mezclados.

\section{METODOLOGÍA}

Las muestras de café tostado y molido se obtuvieron de los supermercados de las provincias de Chiriquí, Veraguas, Coclé y Panamá. Se tomaron muestras al azar y con fecha de expiración posterior al análisis. En el laboratorio, las muestras comerciales con empaque de plástico transparente, se cubrieron con papel aluminio, para protegerlas de la luz y se guardaron a $10^{\circ} \mathrm{C}$, hasta que se analizaron. 


\section{Obtención del extracto acuoso del café}

Para la obtención del extracto acuoso, se utilizó la metodología propuesta por Jeszka-Showron et al. (2015) con modificaciones. Se mezclaron $5 \mathrm{~g}$ de la muestra de café con $100 \mathrm{~mL}$ de agua destilada y se colocaron en una plancha para calentarla hasta alcanzar la temperatura de $90^{\circ} \mathrm{C}$ y mantenerla 5 minutos a esta temperatura. Luego se procedió a filtrar la muestra a través de papel filtro Whatman \#1 y se aforó a $100 \mathrm{~mL}$ con agua. Este extracto fue utilizado para la determinación de contenido de polifenoles totales, contenido de flavonoides y actividad antioxidante.

\section{Determinación del contenido fenólico total en extracto acuoso}

Para la determinación del contenido de polifenoles totales, flavonoides y actividad antioxidante, se utilizó la metodología propuesta por Ayala-Zavala et al. (2012) y Zapata et al. (2013). El extracto de café (50 $\mu \mathrm{L})$, fue mezclado con $3 \mathrm{~mL}$ de agua y $250 \mu \mathrm{L}$ del reactivo Folin-Ciocalteu's $1 \mathrm{~N}$. Se dejó equilibrar por 8 min. Se adicionó $750 \mu \mathrm{L}$ de $\mathrm{Na}_{2} \mathrm{CO}_{3}$ al $20 \%$ y $950 \mu \mathrm{L}$ de agua. Se dejó incubar por 30 min a temperatura ambiente y se procedió a leer en un espectrofotómetro UV/VIS (PG Instruments Ltd, modelo T70+ UV/VIS Spectrometer) a $765 \mathrm{~nm}$. Se preparó una curva de calibración de ácido gálico (ácido 3,4,5-trihidroxibenzoico de Sigma-Aldrich,Co.) con concentraciones de 50, 100, 200, 300, 400, 500 y 1000 ppm, disueltos en agua. Los resultados fueron expresados en mg de equivalentes de ácido gálico por gramo de café (mg de GAE/g).

\section{Determinación de flavonoides en extracto acuoso}

Los flavonoides fueron medidos mezclando $600 \mu \mathrm{L}$ del extracto de café con $2.58 \mathrm{~mL}$ de la solución A (1.8 $\mathrm{mL}$ de $\mathrm{NaNO}_{2}$ al $5 \%$ más $24 \mathrm{ml}$ de agua). Se dejó reposar por 5 min y luego se adicionó $180 \mu \mathrm{L} \mathrm{de} \mathrm{AlCl}_{3}$ al $10 \%$. Se dejó reposar por 1 min. Por último, se adicionó $2.52 \mathrm{~mL}$ de la solución $\mathrm{B}(12 \mathrm{~mL}$ de $\mathrm{NaOH} 1 \mathrm{M}$ más $14,4 \mathrm{ml}$ de agua) y se leyó inmediatamente en un espectrofotómetro UV/VIS (PG Instruments Ltd, modelo T70+ UV/VIS Spectrometer) a $415 \mathrm{~nm}$. Se construyó una curva de calibración de catequina (estándar de Sigma-Aldrich, Co.) con concentraciones de 50, 100, 300, 500, 700 y 1000 ppm, disueltos en agua. Los resultados fueron expresados en $\mathrm{mg}$ de equivalentes de catequina por gramo de café.

\section{Determinación de la actividad antioxidante en extracto acuoso.}

La actividad antioxidante de los extractos de café fue medida en términos de reducción del radical 2,2-difenil-1picrilhidrazilo (DPPH). A $3.9 \mathrm{~mL}$ de solución de DPPH previamente ajustada a absorbancia de 0.700 , se le adicionó $100 \mu \mathrm{L}$ del extracto acuoso de café y se agitó vigorosamente, se dejó incubar por 30 minutos a 27ํㅡ. Luego se leyó en un espectrofotómetro UV/VIS (PG Instruments Ltd, modelo T70+ UV/VIS Spectrometer) a $515 \mathrm{~nm}$. La calibración fue hecha con una solución de trolox (ácido ( \pm )-6-hidroxi-2,5,7,8-tetrametil-croman-2carboxilico de Aldrich) y la actividad antioxidante total fue expresada como milimoles de equivalentes trolox por gramo de café. Se utilizó el metanol para corregir la línea base. La solución de DPPH fue ajustada de la siguiente manera: se preparó una solución de DPPH (2,2-difenil-1-picrilhidrazilo, Sigma-Aldrich, Co.) de concentración 6.59 x 10-5 M y se procedió a ajustar a una absorbancia de 0.7( \pm 0.02$)$, con metanol.

\section{Análisis estadístico}

Los datos de los diferentes parámetros representan las medias y desviaciones estándar de cada una de las muestras por triplicado. Los datos se sometieron a análisis estadísticos como pruebas ANOVA, pruebas de correlación y pruebas de rango como t de student. Todos los datos fueron tratados con el software PAST de acceso gratuito en Internet.

\section{RESULTADOS Y DISCUSIÓN}

En este estudio se analizaron 34 cafés comerciales de Panamá, los cuales son de diferentes variedades, grado de pureza, comercialización y condiciones de cultivo (bajura<900 msnm y altura $>900 \mathrm{msnm}$ ) (ver Figura 1). De los 34 cafés estudiados, el $52.94 \%$ son cafés puros y $47.06 \%$ son mezclas de café y maíz, indicado en el rótulo del paquete del producto. Todos los cafés puros son de las especies $C$. arabica, de las cuales el $66.67 \%$ son de la variedad Arábica $(A)$; el $27.78 \%$, de la variedad Geisha $(G)$ y el $5.55 \%$, de la variedad Pacamara (P). De los cafés puros, el $77.78 \%$ son cultivados en altura (>900 $\mathrm{msnm}$ ) y el $22.22 \%$, en bajura (<900 $\mathrm{msnm})$.

En la Tabla 1, se muestran los resultados del contenido de polifenoles totales, flavonoides y actividad antioxidante de las 18 marcas de cafés puros comerciales de Panamá. En la Tabla, los valores no conectados por la misma letra, en la misma columna, significa que son significativamente diferentes $t=2.02$ $\alpha=0.05$. Las letras son independientes para cada uno de los parámetros evaluados. $A=$ Arábica, $G=G e i s h a$, $\mathrm{P}=$ Pacamara, $\mathrm{CN}=$ Consumo Nacional, E= Exportación. 


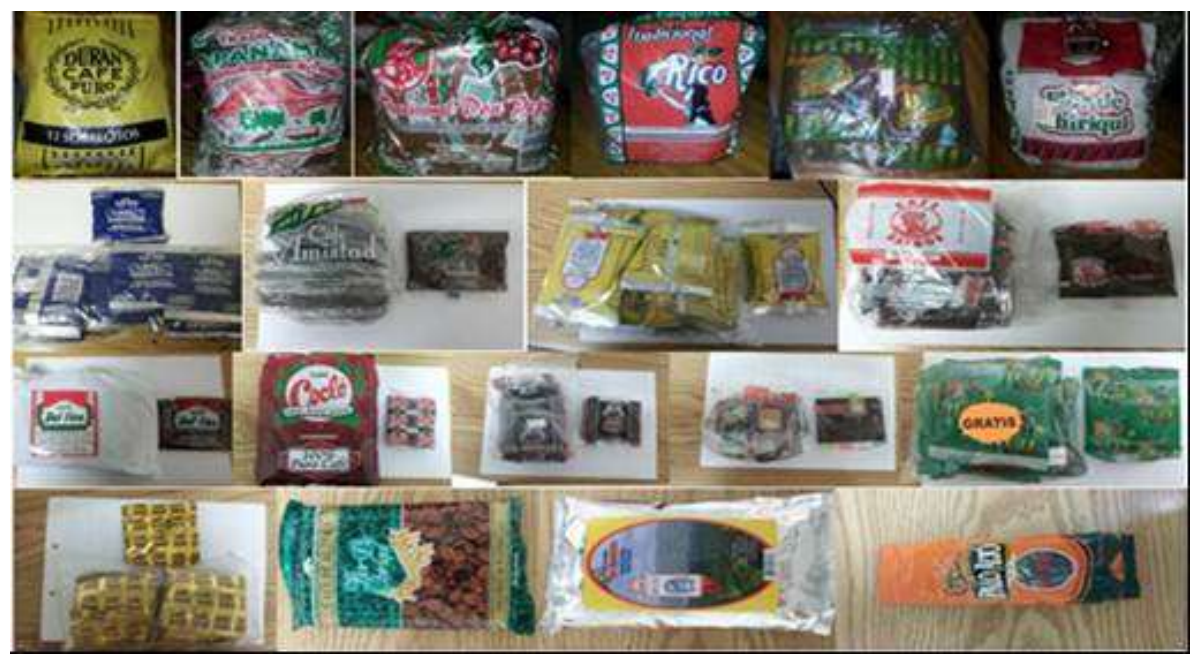

Fig. 1: Muestras de 34 cafés comerciales de Panamá obtenidos en diferentes supermercados del país.

Tabla 1: Contenido de polifenoles totales, flavonoides y actividad antioxidante (extraídos en agua) de las muestras (\#) de cafés puros comerciales de Panamá y la descripción de las mismas.

\begin{tabular}{|c|l|c|l|l|l|c|}
\hline$\#$ & \multicolumn{1}{|c|}{ Variedad } & Uso & $\begin{array}{l}\text { Condición } \\
\text { de Cultivo }\end{array}$ & $\begin{array}{c}\text { Polifenoles totales } \\
(\mathrm{mg} / \mathrm{g})\end{array}$ & $\begin{array}{c}\text { Flavonoides } \\
(\mathrm{mg} / \mathrm{g})\end{array}$ & $\begin{array}{c}\text { DPPH } \\
(\mathrm{mmol} \text { trolox/g) }\end{array}$ \\
\hline 5 & Café arábica & $\mathrm{CN}$ & Altura & $46.82 \pm 1.95 \mathrm{a}$ & $33.71 \pm 0.16 \mathrm{de}$ & $0.183 \pm 0.008 \mathrm{abcd}$ \\
\hline 7 & Café arábica & $\mathrm{CN}$ & Altura & $36.80 \pm 0.60 \mathrm{fg}$ & $29.07 \pm 0.89 \mathrm{~h}$ & $0.166 \pm 0.006 \mathrm{def}$ \\
\hline 8 & Café arábica & $\mathrm{CN}$ & Altura & $34.89 \pm 2.21 \mathrm{~g}$ & $26.22 \pm 0.33 \mathrm{i}$ & $0.156 \pm 0.006 \mathrm{ef}$ \\
\hline 11 & Café arábica & $\mathrm{CN}$ & Altura & $39.46 \pm 1.81 \mathrm{de}$ & $27.85 \pm 0.27 \mathrm{~h}$ & $0.177 \pm 0.005 \mathrm{~cd}$ \\
\hline 14 & Café arábica & $\mathrm{CN}$ & Bajura & $32.65 \pm 1.82 \mathrm{hi}$ & $27.98 \pm 1.13 \mathrm{~h}$ & $0.150 \pm 0.004 \mathrm{fg}$ \\
\hline 15 & Café arábica & $\mathrm{CN}$ & Bajura & $28.91 \pm 0.53 \mathrm{j}$ & $24.70 \pm 0.49 \mathrm{j}$ & $0.138 \pm 0.009 \mathrm{~g}$ \\
\hline 16 & Café arábica & $\mathrm{CN}$ & Bajura & $30.75 \pm 2.44 \mathrm{j}$ & $22.16 \pm 0.64 \mathrm{k}$ & $0.112 \pm 0.013 \mathrm{~h}$ \\
\hline 24 & Café arábica & $\mathrm{CN}$ & Bajura & $44.78 \pm 1.71 \mathrm{ab}$ & $35.32 \pm 0.24 \mathrm{bc}$ & $0.186 \pm 0.002 \mathrm{abc}$ \\
\hline 30 & Café geisha & $\mathrm{E}$ & Altura & $44.99 \pm 1.19 \mathrm{~b}$ & $35.60 \pm 0.56 \mathrm{~b}$ & $0.188 \pm 0.019 \mathrm{abc}$ \\
\hline 31 & Café geisha & $\mathrm{E}$ & Altura & $35.58 \pm 2.38 \mathrm{~g}$ & $32.54 \pm 1.45 \mathrm{ef}$ & $0.175 \pm 0.014 \mathrm{~cd}$ \\
\hline 32 & Café geisha & $\mathrm{E}$ & Altura & $42.85 \pm 1.74 \mathrm{bc}$ & $32.77 \pm 0.09 \mathrm{def}$ & $0.191 \pm 0.005 \mathrm{abc}$ \\
\hline 33 & Café geisha & $\mathrm{E}$ & Altura & $41.59 \pm 1.34 \mathrm{cde}$ & $34.00 \pm 0.55 \mathrm{~cd}$ & $0.200 \pm 0.012 \mathrm{a}$ \\
\hline 34 & Café arábica & $\mathrm{E}$ & Altura & $39.15 \pm 2.52 \mathrm{ef}$ & $30.92 \pm 0.24 \mathrm{~g}$ & $0.177 \pm 0.014 \mathrm{~cd}$ \\
\hline 35 & Café arábica & $\mathrm{E}$ & Altura & $28.60 \pm 1.04 \mathrm{j}$ & $25.37 \pm 0.20 \mathrm{ij}$ & $0.155 \pm 0.006 \mathrm{efg}$ \\
\hline 36 & Café arábica & $\mathrm{E}$ & Altura & $39.48 \pm 0.64 \mathrm{de}$ & $33.55 \pm 0.87 \mathrm{de}$ & $0.182 \pm 0.023 \mathrm{bcd}$ \\
\hline 37 & Café arábica & $\mathrm{E}$ & Altura & $41.76 \pm 1.22 \mathrm{~cd}$ & $31.83 \pm 2.34 \mathrm{fg}$ & $0.169 \pm 0.007 \mathrm{de}$ \\
\hline 38 & Café geisha & $\mathrm{E}$ & Altura & $45.95 \pm 0.93 \mathrm{a}$ & $38.29 \pm 0.30 \mathrm{a}$ & $0.199 \pm 0.001 \mathrm{abc}$ \\
\hline 39 & Café pacamara & $\mathrm{E}$ & Altura & $44.98 \pm 2.73 \mathrm{a}$ & $33.44 \pm 0.49 \mathrm{de}$ & $0.181 \pm 0.006 \mathrm{~cd}$ \\
\hline
\end{tabular}

El contenido de polifenoles totales de las muestras analizadas oscila entre 28.60 y $46.82 \mathrm{mg} \mathrm{GAE} / \mathrm{g}$. Estos valores están dentro del rango de $37-55 \mathrm{mg} / \mathrm{g}$, encontrado en los diferentes tipos de cafés variedad arábica colombianos con tres tipos de tostados y reportados por Pérez-Martínez et al. (2010), además de lo encontrado por Hecimovic et al. (2011) para cafés variedad arábica, cuyo rango es de 25 y $40 \mathrm{mg} / \mathrm{g}$. El contenido de flavonoides de las muestras analizadas se encuentra entre 22.16 y $38.29 \mathrm{mg}$ equivalentes de catequina/g. Estos resultados son más altos que los encontrados por Hecimovic et al. (2011) para cafés variedad arábica, que estuvo en el rango de 15.41 y $17.29 \mathrm{mg} / \mathrm{g}$, dependiendo del grado de tostado. La actividad antioxidante de las muestras analizadas en este estudio, se encuentra entre 0.11 y $0.20 \mathrm{mmol} \mathrm{TE} / \mathrm{g}$. Las muestras de café analizadas en este estudio son de la especie arábica, pero tienen diferentes grados de tostado (información mostrada en el empaque), lo que influye en la variabilidad del contenido de polifenoles totales, flavonoides y actividad antioxidante, como se ha encontrado en otros estudios. Hecimovic et al. (2011), reportó que entre el café arábica con tostado ligero, medio y oscuro, el contenido de polifenoles varió de 25 y $40 \mathrm{mg} / \mathrm{g}$.

En la Tabla 1, se muestran los resultados de la prueba t de student, la cual resultó significativa, con un valor de $t=2.02 \alpha=0.05$ para las diferentes muestras de café puros en los tres parámetros evaluados. Estos 
resultados nos indican que los cafés puros ofrecidos comercialmente en Panamá, tienen diferentes calidades, lo cual se puede deber a que son de diferentes variedades con diferentes condiciones de cultivo y procesamiento. Ello influye en su contenido de sustancias bioactivas y en la actividad antioxidante de las mismas (Decazy et al., 2003; Parras et al., 2007; Hecimovic et al., 2011). Observando los datos de los cafés Geisha, se advierte que hay diferencias significativas entre ellos en los tres parámetros estudiados, a pesar de ser la misma variedad y condición de cultivo; igual sucede con la variedad arábica. Estas diferencias se pueden deber a otros factores que no fueron evaluados en este estudio, como lo son el grado de tostado, tecnología del procesado y el grado de maduración del grano (Parras et al., 2007).

En la Tabla 2, se muestran los resultados del contenido de polifenoles totales, flavonoides y actividad antioxidante de las 16 marcas comerciales de cafés mezclados de Panamá. En la Tabla, los valores no conectados por la misma letra son significativamente diferentes $t=2.02 \quad \alpha=0.05$. Las letras son independientes para cada uno de los parámetros evaluados. El contenido de polifenoles totales de las muestras analizadas está entre 11.17 y $16.10 \mathrm{mg} \mathrm{GAE} / \mathrm{g}$; el contenido de flavonoides, entre 9.36 y 14.92 $\mathrm{mg}$ equivalentes de catequina $/ \mathrm{g}$, y la actividad antioxidante entre 0.025 y $0.061 \mathrm{mmol}$ troloxTE/g. En Miranda (2007) se reporta que las 2 especies que se comercializan en Panamá son Coffea arabica y Coffea canephora, y ambas especies tienen un contenido de polifenoles mayor que lo encontrado en este estudio. Este contenido bajo de polifenoles totales de las marcas de café analizadas en este estudio, se explica porque estos cafés están mezclados con otros granos, como el maíz (dato mostrado en el rótulo del empaque de los cafés e indicado en la Tabla 2), el cual tiene un contenido de polifenoles más bajo que los granos de café. Se analizaron muestras de maíz y su contenido en polifenoles totales fue de $6.53 \mathrm{mg}$ GAE/g (datos no mostrados), lo que explica el bajo contenido de polifenoles de los cafés mezclados con este grano. En la Tabla 2, se muestran los resultados de la prueba t de student, la cual resultó significativa con un valor de $\mathrm{t}=2.02 \alpha=0.05$ en los tres parámetros evaluados para las diferentes muestras de café mezclados. Estos resultados indican que los cafés mezclados ofrecidos comercialmente en Panamá, tienen diferentes calidades, lo cual puede deberse a que las mezclas de café con el maíz tienen diferentes proporciones café/maíz y distintos procesamientos (esta proporción no está indicada en el empaque), lo que influye en su contenido de sustancias bioactivas y la actividad antioxidante de las mismas.

Tabla 2: Contenido de polifenoles totales, flavonoides y actividad antioxidante (extraídos en agua) de las muestras (\#) de cafés mezclados comerciales de Panamá y la descripción de las mismas.

\begin{tabular}{|c|l|c|c|c|c|c|}
\hline$\#$ & Composición & Uso & $\begin{array}{c}\text { Condición de } \\
\text { Cultivo }\end{array}$ & $\begin{array}{c}\text { Polifenoles totales } \\
(\mathrm{mg} / \mathrm{g})\end{array}$ & $\begin{array}{c}\text { Flavonoides } \\
(\mathrm{mg} / \mathrm{g})\end{array}$ & $\begin{array}{c}\text { DPPH } \\
(\mathrm{mmoltrolox} / \mathrm{g})\end{array}$ \\
\hline 1 & Café y maíz & $\mathrm{CN}$ & Bajura & $11.63 \pm 0.34 \mathrm{e}$ & $10.82 \pm 0.44 \mathrm{defg}$ & $0.042 \pm 0.002 \mathrm{bcd}$ \\
\hline 2 & Café y maíz & $\mathrm{CN}$ & Bajura & $11.17 \pm 0.44 \mathrm{e}$ & $10.50 \pm 0.28 \mathrm{efg}$ & $0.038 \pm 0.004 \mathrm{cdef}$ \\
\hline 3 & Café y maíz & $\mathrm{CN}$ & Bajura & $13.31 \pm 0.60 \mathrm{bcde}$ & $13.81 \pm 0.22 \mathrm{abc}$ & $0.037 \pm 0.001 \mathrm{cdef}$ \\
\hline 4 & Café y maíz & $\mathrm{CN}$ & Bajura & $12.24 \pm 0.59 \mathrm{de}$ & $12.21 \pm 0.29 \mathrm{bcdef}$ & $0.038 \pm 0.010 \mathrm{cdef}$ \\
\hline 6 & Café y maíz & $\mathrm{CN}$ & Bajura & $13.57 \pm 0.14 \mathrm{bcde}$ & $10.07 \pm 0.43 \mathrm{fg}$ & $0.040 \pm 0.004 \mathrm{bcde}$ \\
\hline 10 & Café y maíz & $\mathrm{CN}$ & Bajura & $12.91 \pm 0.47 \mathrm{bcde}$ & $12.55 \pm 1.78 \mathrm{bcdef}$ & $0.042 \pm 0.006 \mathrm{bcd}$ \\
\hline 13 & Café y maíz & $\mathrm{CN}$ & Bajura & $13.27 \pm 0.37 \mathrm{bcde}$ & $10.26 \pm 1.91 \mathrm{efg}$ & $0.035 \pm 0.006 \mathrm{cdef}$ \\
\hline 17 & Café y maíz & $\mathrm{CN}$ & Bajura & $15.28 \pm 0.63 \mathrm{ab}$ & $14.81 \pm 0.43 \mathrm{ab}$ & $0.025 \pm 0.001 \mathrm{f}$ \\
\hline 20 & Café y maíz & $\mathrm{CN}$ & Bajura & $14.83 \pm 0.60 \mathrm{abc}$ & $11.81 \pm 0.29 \mathrm{cdefg}$ & $0.037 \pm 0.004 \mathrm{cdef}$ \\
\hline 21 & Café y maíz & $\mathrm{CN}$ & Bajura & $11.60 \pm 0.99 \mathrm{de}$ & $11.08 \pm 1.11 \mathrm{abcd}$ & $0.040 \pm 0.004 \mathrm{bc}$ \\
\hline 22 & Café y maíz & $\mathrm{CN}$ & Bajura & $15.49 \pm 1.33 \mathrm{ab}$ & $13.49 \pm 1.39 \mathrm{abcd}$ & $0.053 \pm 0.003 \mathrm{ab}$ \\
\hline 25 & Café y maíz & $\mathrm{CN}$ & Bajura & $12.61 \pm 1.54 \mathrm{cde}$ & $9.36 \pm 0.54 \mathrm{~g}$ & $0.048 \pm 0.002 \mathrm{abc}$ \\
\hline 26 & Café y maíz & $\mathrm{CN}$ & Bajura & $16.10 \pm 0.37 \mathrm{a}$ & $14.71 \pm 0.90 \mathrm{ab}$ & $0.061 \pm 0.005 \mathrm{a}$ \\
\hline 27 & Café y maíz & $\mathrm{CN}$ & Bajura & $12.55 \pm 0.35 \mathrm{cde}$ & $13.59 \pm 0.67 \mathrm{abc}$ & $0.026 \pm 0.002 \mathrm{ef}$ \\
\hline 28 & Café y maíz & $\mathrm{CN}$ & Bajura & $14.57 \pm 0.97 \mathrm{abcd}$ & $12.91 \pm 0.17 \mathrm{abcde}$ & $0.035 \pm 0.003 \mathrm{cdef}$ \\
\hline 29 & Café y maíz & $\mathrm{CN}$ & Bajura & $14.83 \pm 1.20 \mathrm{abc}$ & $14.92 \pm 0.87 \mathrm{a}$ & $0.030 \pm 0.008 \mathrm{def}$ \\
\hline
\end{tabular}

En la Fig. 2, se muestra el gráfico entre el contenido de polifenoles totales (PFT) y la actividad antioxidante de los cafés puros comerciales de Panamá analizados, y se encontró una correlación de $R^{2}=0.69$. Este resultado nos permite inferir que los PFT son componentes del café que aportan un porcentaje importante a la capacidad antioxidante del mismo. Los PFT junto con otros componentes (Pérez-Hernández et al., 2012) le confieren al café la propiedad de ser considerado un producto con alto poder antioxidante. Los cafés puros estudiados son de la especie arábica y si hacemos una comparación con lo informado por otros estudios, observamos que la correlación obtenida en éste, es menor. Por ejemplo, se encontró una 
correlación de $\mathrm{R}^{2}=0.85$ entre el contenido de polifenoles totales y la actividad antioxidante en muestras de cafés comerciales de Rumanía (Trandafir et al., 2013). Igualmente, una alta correlación $\left(R^{2}=0.97\right)$ fue observada entre los polifenoles totales y la actividad antioxidante para cafés arábica y robusta, procesados (Pérez-Hernández et al., 2012), así como una correlación de $\mathrm{R}^{2}=0.89$ entre el contenido de polifenoles totales y la actividad antioxidante ABTS del residuo del café geisha de Panamá (Shang et al., 2017). Esto puede deberse a que el grado de tostado es diferente entre estas muestras y las muestras de otros estudios.

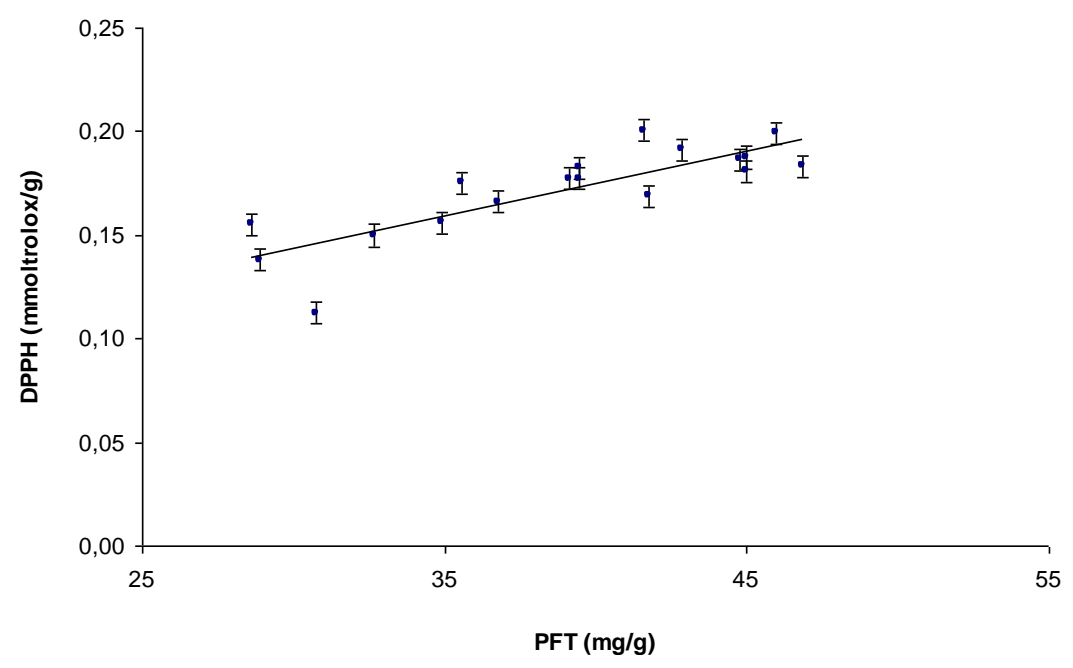

Fig. 2: Correlación entre el contenido de polifenoles totales $(\mathrm{mg} / \mathrm{g})$ y actividad antioxidante en $\mathrm{mmol}$ trolox (TE)/g de las muestras de café puros. $R^{2}=0.69, \alpha=0.05$.

En la Fig. 3, se muestra el gráfico entre el contenido de polifenoles totales y la actividad antioxidante de los cafés mezclados comercializados en Panamá, y se encontró una correlación de $\mathrm{R}^{2}=0.040$. Este resultado nos sugiere que al mezclar el café con el maíz, ocurre una disminución de la actividad antioxidante. Estas muestras de café mezclados con otros granos tienen otros compuestos con actividad antioxidante menor que el café y también tienen bajo contenido de polifenoles totales, como es el caso de los granos de maíz.

En la Fig. 4 se muestra que los cafés de la especie Coffea arabica var. geisha son los que tienen mayor contenido de polifenoles totales (35.58 a $45.95 \mathrm{mg} / \mathrm{g})$ y actividad antioxidante $(0.175$ a $0.200 \mathrm{mmol}$ trolox $(\mathrm{TE}) / \mathrm{g})$. No hay reporte de análisis fisicoquímico de los granos de café geisha, ya que no se había comercializado antes; y es en Panamá, donde se ha comercializado. En Shang et al. (2017), se reportó el contenido de polifenoles $(21.01 \mathrm{mg} / \mathrm{g})$ y actividad antioxidante $(0.06 \mathrm{mmol}$ trolox(TE)/g) del residuo sólido del café geisha, pero no del grano. Sin embargo, se observa que este residuo tiene una cantidad importante de polifenoles totales, superior al contenido de los cafés mezclados de este estudio. Los resultados que se muestran en esta Figura, son de gran relevancia e interés, ya que son los primeros obtenidos del extracto acuoso del café molido, variedad Geisha y, por ende, pueden servir como línea base para realizar futuros estudios acerca de las propiedades de esta variedad de café, que está adquiriendo auge comercial a nivel internacional.

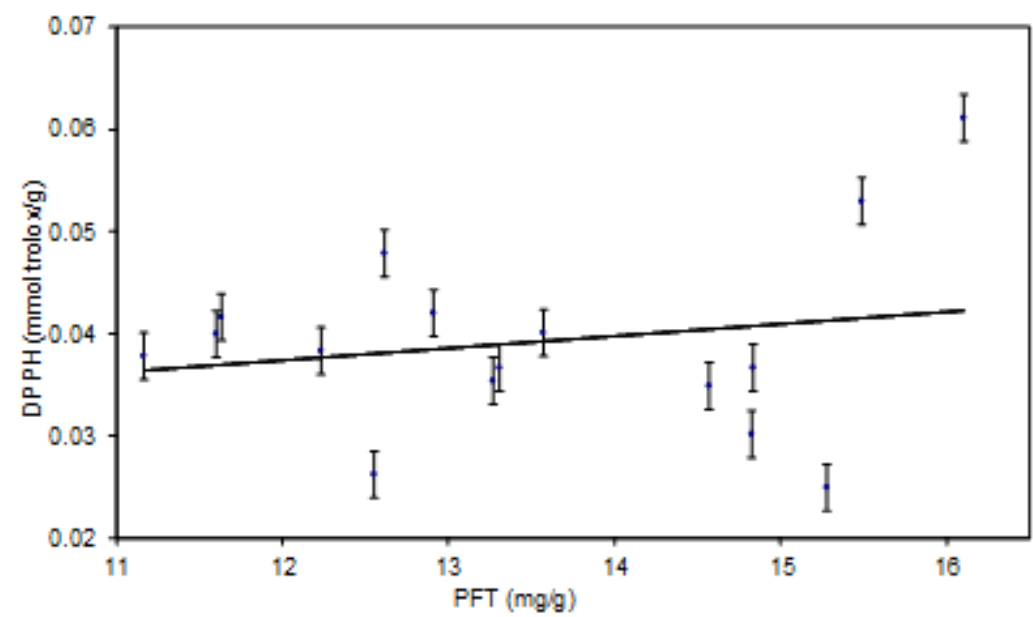

Fig. 3: Correlación entre el contenido de polifenoles totales $(\mathrm{mg} / \mathrm{g})$ y actividad antioxidante en $\mathrm{mmol}$ trolox $(T E) / g$ de las muestras de café mezclado con otros granos. $R^{2}=0.040, \alpha=0.05$. 


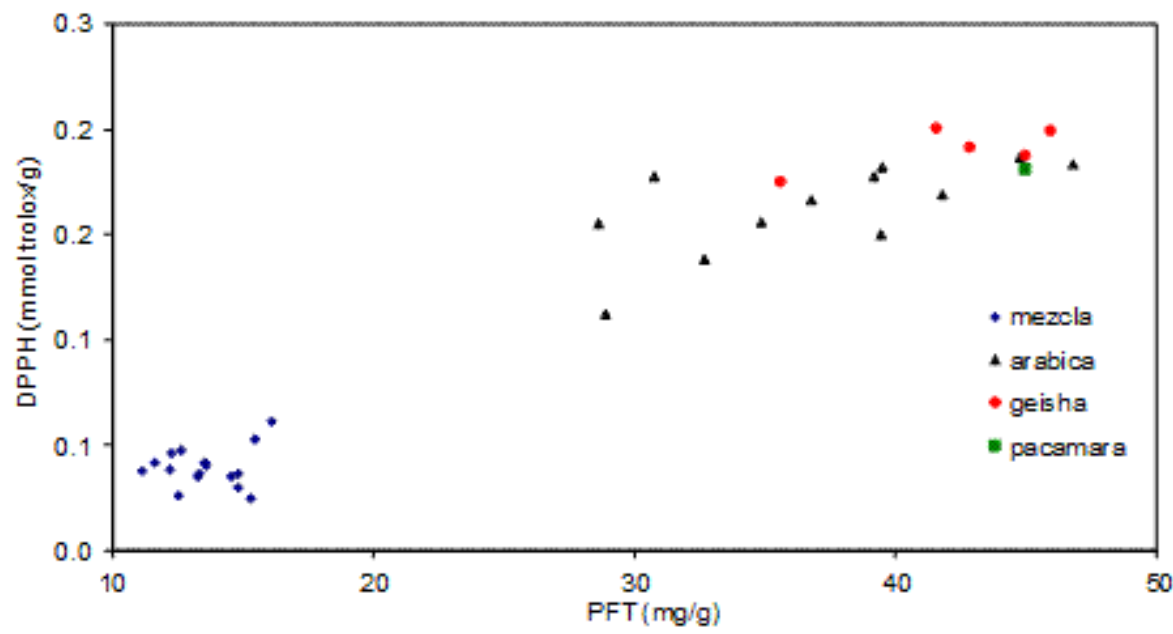

Fig. 4: Gráfico de dispersión del contenido de polifenoles totales (PFT mg GAE/g) vs actividad antioxidante (DPPH mmol trolox(TE)/g), según la variedad del café.

En la Fig. 5, se muestra que los cafés que son cultivados a alturas de más de $900 \mathrm{msnm}$ son los que tienen mayor contenido de polifenoles totales y de actividad antioxidante, lo cual concuerda con los resultados obtenidos por (Guyot and et al., 1996; Decazy et al., 2003) en los cuales se muestra la influencia positiva de la altitud en la calidad del café arábica, debido a la variación en la fisiología de la planta en cuanto al tiempo de maduración del grano, así como a la acidez y aroma que alcanza.

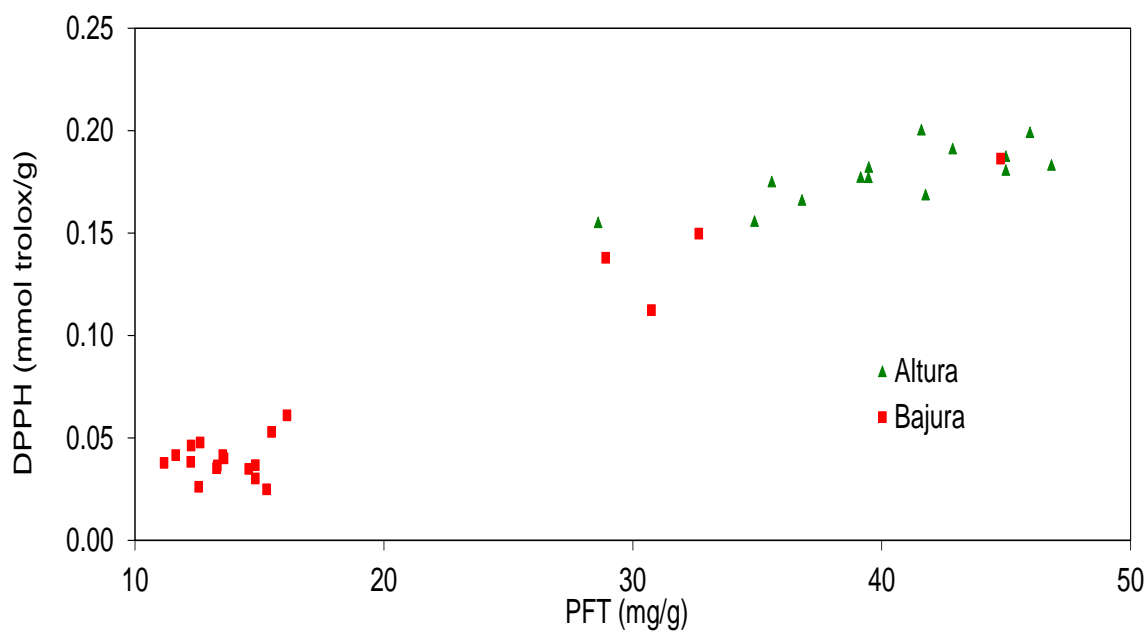

Fig. 5: Gráficos de dispersión del contenido de polifenoles totales (PTF mg GAE/g) vs la actividad antioxidante (DPPH mmol trolox (TE)/g) según las condiciones de cultivo.

\section{CONCLUSIONES}

Los datos obtenidos en este trabajo, muestran que los cafés comerciales de Panamá tienen un contenido de polifenoles totales (PFT), flavonoides y actividad antioxidante que varían en un amplio rango. En este país, se comercializan marcas de café cuyo contenido es puro, pero también otros mezclados con granos, como el maíz. La mayoría de los cafés de consumo nacional son mezclas de la especie Robusta, Arábica y maíz, mientras que los cafés de exportación son de la especie Arábica. Los cafés puros tienen el mayor contenido de sustancias bioactivas; de ellos, la variedad Geisha presenta alto contenido de estas sustancias, dato importante, debido al auge comercial de la misma. Se encontró correlación $\left(R^{2}=0.69\right)$ entre el contenido de polifenoles totales y la actividad antioxidante de los cafés puros; este resultado nos permite inferir que los PFT son componentes del café que aportan un porcentaje importante a la capacidad antioxidante de este tipo de bebida.

\section{AGRADECIMIENTOS}

Los autores agradecen a la Vicerrectoría de Investigación y Posgrado de la Universidad Autónoma de Chiriquí VIP-UNACHI y al Sistema Nacional de Investigación de la Secretaría Nacional de Ciencia Tecnología e Innovación de Panamá SNI - SENACYT. También agradecen al Ing. Alexis Bonilla del 
Ministerio de Desarrollo Agropecuario (MIDA), por su colaboración en las diferentes actividades y a los propietarios, administradores y técnicos de los Beneficios de Café encuestados, por la información y muestras suministradas.

\section{REFERENCIAS}

Ayala-Zavala, J.F.; A.B. Silva-Espinoza; R.M. Cruz-Valenzuela; M.A. Villegas-Ochoa; M. Esqueda; G.A. González-Águila y Y. Calderón-López, Antioxidant and antifungal potential of metanol extracts of Phenillus spp. From Sonora, Mexico, Rev. Iberoam. Micol., 29(3), 132-138 (2012)

Decazy, F.; J. Avelino; B. Guyot; J.J. Perriot; C. Pineda y C. Cilas, Quality of different Honduran coffees in relation to several environments, J. Food Sci., 68(7), 2356-2361 (2003)

Esquivel, P. y V. M. Jiménez, Functional properties of coffee and coffee by-products, Food Res. Int., 46, 488-495 (2012)

Farah, A.; M.C. Monteiro; V. Calado; A.S. Franca y L.C. Trugo, Correlation between cup quality and chemical attributes of Brazilian coffee, Food Chem. 98, 373-380 (2006)

Farah, A., 2 Coffee Constituents, in Coffee: Emerging Health Effects and Disease Prevention by Ch. YiFang, $1^{\text {a }}$ Ed., pp 21-58, John Wiley \& Sons, Inc., U.S.A. (2012)

Gallignani, M.; M. Torres; C. Ayala y M.R. Brunetto, Determination of caffeine in coffee by means Fourier transform infrared spectrometry, Rev. Téc. Ing. Univ. Zulia, 31(2), 159-168 (2008)

Guyot, B.; D. Gueule; J.C. Manez; J.J. Perriot; J. Giron y L. Villain, Influence de l'altitude et de l'ombrage sur la qualité des cafés Arábica, Plant Rech. Dévelop, 3, 272-283 (1996)

Hečimović, I.; A. Belščak-Cvitanović; D. Horžić y D. Komes, Comparative study of polyphenols and caffeine in different coffee varieties affected by the degree of roasting, Food Chem., 129, 991-1000 (2011)

Jeszka-Skowron, M.; A. Zgola-Grzeskowiak y T. Grzeskowiak, Analytical methods applied for the characterization and the determination of bioactive compounds in coffee, Eur Food Res. Technol., 240, 19-31 (2015)

Kocadağlı, T. y V. Gökmen, Effect of roasting and brewing on the antioxidant capacity of espresso brews determined by the QUENCHER procedure, Food Res. Int., doi:10.1016/j.foodres.2016.03.004 (2016)

Ludwig, I.A.; L. Sánchez; B. Caemmerer; L.W. Kroh; M. P. De Peña y C. CID, Extraction of coffee antioxidants: Impact of brewing time and method, Food Res. Int., 48, 57-64 (2012)

Ludwing, I.A.; P. Mena; L. Calani; C. Cid; D. Del Río; M.E.J. Lean y A. Crozier, Variations in caffeine and chlorogenic acid contents of coffees: what are we drinking?, Food Funct., 5, 1718-26 (2014)

Martin, M. J.; F. Pablos y A.G. González, Characterization of green coffee varieties according to their metal content, Anal. Chim. Acta, 358, 177-183 (1998)

Ministerio de Desarrollo Agropecuario, Información Estadística sobre la exportación y producción del rubro: café, años cafetaleros 1980-81 al 2012-13, Panamá (2013)

Miranda, A., Plan estratégico para el café 2007-2016. Ministerio de Desarrollo Agropecuario de Panamá, pp. $81(2007)$

Nam, V., Informe del mercado de café Marzo 2015, Demanda se mantiene dinámica a pesar de otra caída del mercado de café, $1-5$ (2015)

Parras, P.; M. Martineztome; A. Jimenez y M. Murcia, Antioxidant capacity of coffees of several origins brewed following three different procedures, Food Chem., 102, 582-592 (2007)

Pérez-Hernández, L.M.; K. Chávez-Quiroz; L. Á. Medina-Juárez y N. Gámez Meza, Phenolic Characterization, melanoidins, and Actioxidant activity of some Commercial Coffees from Coffea arabica and Coffea canephora, J. Mex. Chem. Soc., 56(4), 430-435 (2012)

Shang, Y.-F.; J.-L. Xu; W.-J. Lee y B.-H. Um, Antioxidative polyphenolics obtained from spent coffee grounds by pressurized liquid extraction, South African Journal of Botany, 109, 75-80 (2017) 
Trandafir, I.; V. Nour y M. E. Ionica, Antioxidant capacity, phenolic acids and caffeine contents of some commercial coffees available on the Romanian market, Arch. Latinoam. Nutr., 63, 87-94 (2013)

Vega, A.; S. Reyes; J. De León; A. Bonilla y H. Franco, Cuantificación de cafeína en cafés comerciales de Panamá, 30, 57-64 (2014)

Vega, P., Café para el gusto exigente. Publicidad y consumo de café en Costa Rica 1900-1930, Revista Historia, 55-56, 73-98 (2007)

Vignoli, J.A.; D.G. Bassoli y M.T. Benassi, Antioxidant activity, polyphenols, caffeine and melanoidins in soluble coffee: The influence of processing conditions and raw material, Food Chem., 124, 863-868 (2011)

Wanyika, H.; E. Gatebe y L. Gitu, Determination of caffeine content of tea and instant coffee brands found in the Kenyan market, African J. Food, 4, 353-358 (2010)

Zapata, K.; F.B. Cortés y B.A. Rojano, Polifenoles y Actividad Antioxidante del Fruto de Guayaba Agria (Psidium araca), Información Tecnologica, 24(5), 103-112 (2013) 
